Editorial

\title{
Healthcare Big Data Management and Analytics in Scientific Programming
}

\author{
Shah Nazir ${ }^{(D},{ }^{1}$ Iván García-Magariño $\left(\mathbb{D},{ }^{2}\right.$ Rodziah Binti Atan, ${ }^{3}$ and Shaukat Ali ${ }^{4}$ \\ ${ }^{1}$ Department of Computer Science, University of Swabi, Swabi, Pakistan \\ ${ }^{2}$ Department of Software Engineering and Artificial Intelligence, Complutense University of Madrid, 28040 Madrid, Spain \\ ${ }^{3}$ Faculty of Computer Science and Information Technology, University Putra Malaysia, Selangor, Malaysia \\ ${ }^{4}$ Department of Computer Science, Islamia College, Peshawar, Khyber Pakhtunkhwa, Pakistan \\ Correspondence should be addressed to Shah Nazir; shahnazir@uoswabi.edu.pk
}

Received 28 July 2021; Accepted 28 July 2021; Published 15 October 2021

Copyright (C) 2021 Shah Nazir et al. This is an open access article distributed under the Creative Commons Attribution License, which permits unrestricted use, distribution, and reproduction in any medium, provided the original work is properly cited.

The systems of healthcare are being transmuted by scientific improvements in the information of medical systems, electronic records in medical, smart, and wearable devices, and handheld devices. This growth in medical big data, together with the expansion of computational approaches in the area of healthcare, has aided researchers and practitioners to excerpt and visualize medical big data in a novel scale of research. The role of scientific programming in providing solutions to existing and forthcoming issues exists in the organization of large-scale data in healthcare, such as by assisting in the handing out of vast data volumes, modelling of composite systems, and sourcing derivations from healthcare data and simulations. Visualization is a significant tool in producing diagrams, images, or animations to transfer healthcare messages and improve understandings. Programming tools, including Apache Hadoop, Informatica PowerCenter, and Tableau, analyze data extremely efficiently and enable the visualization of meaningful insights extracted from big data.

Research is needed that explores the integration of big data and healthcare from a scientific programming perspective. Research that considers technological and computational barriers to big data management has clear applications in the area of big data in healthcare. Diverse approaches have been in practice by researchers and practitioners. These areas include decision support systems for big data in healthcare, programming for medical big data visualization and its representation, applications of machine and/or deep learning algorithms and applications in big data analytics for healthcare, data warehouse and knowledge representation in healthcare technologies, data mining in Internet of Things (IoT) and healthcare systems, and probabilistic computing approaches to the management of big data in healthcare.

The papers included in this Special Issue cover the details of the scientific aspects which are mainly involved in the field of healthcare big data management, its organization, and the role of programming to deal with a particular situation of big data. Raji et al. used the wavelet transform performance against future wireless application system requirements and offered guidelines and methods for wavelet applications in $5 \mathrm{G}$ waveform design. The detailed effect of healthcare was targeted. With the help of images as test data, a detailed performance comparison of the Fourier transform and various wavelet transforms have been done with the help of modulation and demodulation complexity, energy efficiency, latency, reliability, spectral efficiency, robustness to time and frequency selective channels, and effect of transmission and reception considered as the key performance indicators. After this, the guidelines are presented for the wavelet transform. Mansoor et al. present an overview of the data acquisition, feature extraction, and classification algorithm approaches adopted by researchers and practitioners in the previous years. Some classification algorithms for EEG-based BCI systems are adaptive classifiers, tensor classifiers, transfer learning approach, and deep learning, as well as some miscellaneous approaches. From the experimental results of the method, it was concluded that using the 
adaptive classifiers, precise results were obtained compared to the static classification approach. Deep learning algorithms were adopted for developing and achieving the specified objectives and implementation.

R. Khan et al. investigated the protection level offered by UUP. The query profile distance attack as machine learningbased attack was presented for evaluating the effectiveness of UUP in privacy protection. The approach demonstrates the distance between upcoming query and the user profile. Ten classification algorithms were used for the experimental setup. These algorithms include the tree-based, lazy learner, rule-based, metaheuristic, and Bayesian families for the sake of comparison. Two subsets of an American online dataset including noisy and clean datasets were used for experimental process. Results of the experiments showed that the suggested approach links more than $70 \%$ queries to the right users with $72 \%$ precision for the clean dataset, while for the noisy dataset, the proposed approach associates more than $40 \%$ queries to the right user with a precision of $70 \%$.

Raja et al. presented a systematic literature review of big data related to healthcare. The study evaluated 34 journal articles for the years 2015 to 2019 accordingly to the defined inclusion and exclusion criteria. The study specified the research in the area of big data with its applications and challenges in healthcare implementation. Kamran Ul haq et al. analyzed the studies based on the approach of big data in mental illness and treatment. Diverse types of mental illness such as bipolar disorder, personality disorder, and depression are discussed. The user behavior based on mental illness for drug addiction and suicide are highlighted. An overview of the approaches and tools for predicting the mental condition of a patient based on machine learning and artificial intelligence is given. Hu et al. presented an approach of information research for fusing a large volume of heterogeneous data produced by a charging pile resultant to the new energy electric vehicle in the network of vehicles and present the concept of cloud computing as a module of storage for facilitating the storage and associated expansion of big data. Khan et al. identified 26 exercises of gym from the literature. Out of these, 14 were unique and 12 were common in the existing literature. The study also finds the suitable smartphone attachment position and the number of sensors for predicting exercise with high accuracy.

$\mathrm{N}$. Boudjellal et al. presented an approach of distant supervision for relation extraction, providing a generic architecture of this task based on the existing approaches. The study reviewed the approaches used in the literature targeting the current areas of research with details of knowledge bases used in the process along with the corpora which can be supportive for trainee practitioners looking for knowledge in the field. Feng et al. proposed a framework of fogbased IoT healthcare for reducing the consumption of energy of fog nodes. The results of the study show that the performance of the suggested framework is effective in terms of the network delay and usage of energy. Discussions and suggestions are made for the services of big data for fog devices and analytics of healthcare big data are presented.

\section{Conflicts of Interest}

The editors declare no conflicts of interest.

Shah Nazir

Iván García-Magariño

Rodziah Binti Atan

Shaukat Ali 